\title{
Adolescence et cancer : deux crises pour le prix d'une...
}

\author{
M.-F. Bacqué \\ (C) Springer-Verlag France 2009
}

L'adolescence est la période exemplaire de la vie dans laquelle le duel des pulsions de vie et de mort atteint un sommet. Les pulsions de vie sont exacerbées grâce à ce corps « tout neuf » qui sort de l'enfance, désirable en diable, ou plus simplement mû par un désir qui transsude au-delà des défenses parfois les plus massives. Les pulsions de mort s'expriment dans la résistance à ce désir d'aller vers les autres, cet enfouissement derrière la lourde frange teinte en noir corbeau, sans cesse rabattue sur les yeux. Ce corps replié, figé dans un coin, cette allure traînante qui témoigne de la fatigue ou du manque de tonus appartiennent encore au jeune de 14-15 ans qui a " tout vu », « tout connu » et pour qui le monde est déjà d'une routine effrayante. Ici, l'adolescent reste prostré, «protégé » par son téléphone portable, sa PlayStation et son lecteur mp3 sur les oreilles, replié sur lui-même, là, il est rivé à son miroir sur lequel il détaille inlassablement les nouveaux boutons d'acné ou les cernes liés au manque de sommeil. Ces caricatures d'adolescents, nous les retrouvons à l'hôpital, dans les services de pédiatrie. Au départ, les « grands » se départissent soigneusement des enfants, auxquels ils n'apprécient pas d'être assimilés, puis, progressivement, alors que les traitements réduisent en miettes leur allure, leur style savamment élaborés de jadis, ils s'ouvrent aux autres et maternent les plus petits.

\section{Souffrance adolescente}

Oui, les adolescents souffrent, et cette souffrance est peutêtre la plus difficile à accompagner nonobstant le déni général qu'ils préfèrent lui apposer. En dehors d'une maladie grave, l'adolescence consiste à supporter d'avoir un corps sexué d'adulte avec une maturité d'enfant. L'adolescence consiste aussi à tolérer et à dépasser la perte de l'enfance. Cette perte concerne bien sûr les avantages de l'enfance : la pensée magique et la toute-puissance en font partie, mais aussi l'amour infantile porté à des parents protecteurs en

M.-F. Bacqué $(\bowtie)$

Département de psychologie, université Louis-Pasteur,

12, rue Goethe, F-67000 Strasbourg, France

e-mail : mfbacque@club-internet.fr toute circonstance, exerçant le pardon ou tentant sans cesse la conciliation. La maladie du cancer vient littéralement questionner ce conflit : renoncer à la toute-puissance infantile au profit de la « raison raisonnable » des adultes. Mais il y a pire, reconnaître l'impuissance de ses parents, ceux qui ont été tenus pour des héros depuis la naissance. Paradoxalement, l'adolescent doit affronter les poussées d'énergie qui provoquent la révolte contre l'absence de « justice » de cette fatalité qui le frappe, il doit accepter de reconnaître sa détresse lors de moments de vide, de solitude. Il sait qu'il faudra trouver une nouvelle identité, alors qu'il préférerait se fondre dans la masse. Le jeune redoute, plus que tout au monde, de se montrer faible et confus, alors que son corps témoigne d'une maturité physique évidente. Il se complaît encore dans ce qu'il estime ses privilèges (ceux de la jeunesse), comme la liberté, l'anticonformisme et la provocation. Il les brandit parfois comme le triste étendard de ses capacités antérieures déchues. Car il est honteux d'éprouver la tristesse et surtout la dépendance affective...

\section{Crise adolescente et incapacité à se conformer au traitement}

Point d'étonnement alors à constater le manque d'observance des adolescents (qu'ils soient diabétiques ou cancéreux d'ailleurs). Perdus dans leur mouvement intérieur, ils peuvent difficilement absorber un nouveau changement, un autre défi aussi grave, celui de suivre un traitement pour la vie peut-être... Pourquoi, en effet, les cancers de l'adolescent guérissent relativement moins bien que ceux des enfants ? À leur propre ambivalence à l'égard de la mort qui rôde, répond en effet l'ambivalence des parents. Car l'adolescence ne laisse pas les parents indemnes. Or, ceux qui pensaient avoir franchi l'étape la plus rude sont brutalement rejetés en arrière par la maladie qui réamorce la dépendance de l'adolescent désormais aussi passif qu'un bébé.

Un adolescent malade ? Et c'est le couple parental qui se confronte au désastre. Si l'enquête JADE menée à l'institut Gustave-Roussy et à Curie montre que mère et adolescent sont congruents dans la perception de la détresse, ce couple nouvellement reformé met à mal celui plus officiel des parents qui croyaient retrouver un peu de liberté... 
Certains parents abandonnent leur ado qui s'accroche aux soignants pour compenser... D'autres au contraire s'enferrent dans la régression de leur jeune redevenu leur « toutpetit $» .$. Les autres suivent, tant bien que mal, cachant leurs larmes, se cachant parce que leur fils ou leur fille ne les trouve pas assez «stylés » ou encore disparaissant parce qu'ils ne supportent plus la tension engendrée par ce malade trop singulier... Tout cela contribue, à n'en pas douter, une part causale dans la difficulté à observer les prescriptions médicales de plus de $50 \%$ des adolescents.

Quand un ado est malade, la famille a encore plus d'importance que pour un malade plus âgé.

\section{Un accompagnement qui prend en compte la dynamique de l'adolescence}

Le congrès de la société française de psycho-oncologie offre une approche multipartite qui permet de dépasser l'angoisse de la crise et d'apporter des idées neuves. Les caractéristiques de l'adolescence nous persuadent de la singularité de cet être en devenir qui doit accepter d'être « différemment le même » et qui s'en protège par un comportement parfois autistique. La séduction de ce jeune, tout comme sa fragilité, pourraient nous conduire à un maternage qu'il rejette le plus souvent. Paradoxalement, le traiter comme un adulte peut le faire plonger dans la dépression. Quoi de plus vertigineux en effet qu'une vie qui s'annonce longue, mais déjà grevée de séquelles, de stérilité potentielle, de handicap ou d'invalidité ?

On rétorquera que les guérisons surviennent dans près de $50 \%$ des cas, mais le prix est lourd pour toute la cellule familiale.

Ici s'interpose peut-être le groupe des adolescents. On sait combien, depuis les travaux des sociologues sur les bandes de jeunes, le groupe sert de support identificatoire et d'alter ego substitutif. Les services de cancérologie pourraient, en lien avec les associations (merci à Jeunes Solidarité Cancer [JSC] pour sa présence, ses blogs, son site Internet), développer plus systématiquement les groupes d'adolescents, autour de la révélation du cancer, pendant les traitements, mais surtout après la guérison. Ils sont nombreux ces jeunes adultes qui ne savent plus qui consulter pour parler des effets secondaires tardifs de leur cancer : pédiatrie ? Ils n'ont plus l'âge de jouer dans les couloirs... Oncologie adulte ? Ils ne supportent pas cette indifférence... Des forums à la Ligue contre le cancer, des chats sur le site de JSC, des SMS sur les téléphones portables pour rappeler une présence, ce sont tous ces moyens, largement développés, qui permettront un maintien du lien, plus que l'austère, mais sans doute bien nécessaire, "surveillance sanitaire » que les pouvoirs publics appellent de leurs vœux... Échanger avec les jeunes, les parents permettra à ces Journées des 5 et 6 novembre 2009 de répertorier et de mettre en œuvre les idées les plus adéquates pour que l'adolescence reste une étape dans la vie et pas un écueil... 